\title{
Modified Golomb-Rice Algorithm for Color Image Compression
}

\author{
Mr. Rahul Sharma \\ Auricle Technologies Bikaner Rajasthan India \\ rahulmnul@gmail.com
}

\begin{tabular}{|c|c|}
\hline $\begin{array}{l}\text { Article History } \\
\text { Article Submission } \\
2 \text { December } 2012 \\
\text { Revised Submission } \\
17 \text { February } 2012 \\
\text { Article Accepted } \\
15 \text { March } 2012 \\
\text { Article Published } \\
31^{\text {st }} \text { March } 2013\end{array}$ & $\begin{array}{l}\text { Abstract } \\
\text { The memory required to store the color image is more. We have reduced the memory } \\
\text { requirements using Golomb-rice algorithm. Golomb-rice algorithm consists of the } \\
\text { following two steps. In Golomb-Rice algorithm the first step is to compress the image } \\
\text { using discrete wavelet transform. By using DWT compression the } 8 \times 8 \text { image is } \\
\text { converted into } m \times n \text { sub-windows and it is converted into raster file format for } \\
\text { producing } m \times n-1 \text { differential data. Encoding is done by using Golomb-Rice coding. } \\
\text { After encoding, the process length, code word and size are calculated by using GR } \\
\text { coding.In the second step decoding is done by GR coding based on the obtained } \\
\text { length and code word. After that decoded image is decompressed in order to get the } \\
\text { original image by using the inverse discrete wavelet transform. } \\
\text { Keywords: } D W T, \text { IDWT, Golomb-Rice algorithm [GR], code length. }\end{array}$ \\
\hline
\end{tabular}

\section{Introduction}

Images are of two types. One is gray scale Image and another one is color image. On comparison with the gray scale image, more minute details can be observed in the color image. Efficient memory and bandwidth reduction is achieved through lossless compression and decompression standards of color images. The compression and decompression techniques are achieved by two mechanisms [1][2] i.e. Differential data conversion using raster file and GR algorithm.

In the existing method, image of size $\mathrm{m} \mathrm{x} \mathrm{n}$ is taken as an input and it is converted into differential data by using DDPCM which will produce image of size $\mathrm{m} \times \mathrm{n}-1$. Then the previous output has been given as an input to the compression block. In the compression block the image is compressed and it will sent to the GR encoder block we can check whether the image is compressed or not by using $\mathrm{v}$ bit [3]. If $\mathrm{v}$ bit is 0 , then the image is not compressed and if $v$ bit is 1 , means the image is compressed based on $v$ bit only, we can encode the image by using GR encoder, based on the code word, quotient and remainder obtained. The output of the GR encoder has been given as an input to the decoder block, in the decoder block the encoded image has been decoded by using GR decoder [4]. Then decoded image is decompressed by using inverse DDPCM and then original image has been obtained [5].

\section{Related Work}

The discrete choose of compression algorithm plays a vital role in memory architectures because the original images are reconstructed from the image which is already compressed. Hardware compression methodologies are preferred than software compression as it supports implementation of real time features in case of data processing. Various algorithms like Ziv code, Golomb Rice code, Calic code and JPEG-LS code were proposed for the sake of loseless image compressing standards. Mostly code, Golomb Rice code, Calic code and JPEG-LS code supports software based image compression standards with high compression rates thereby requiring complex hardware implementations[6][7].

Many researchers have proposed modern memory architectures to improve the efficacy of embedded systems through dedicated image compression techniques [8]. Various architectures like OS based memory compression; DCT based image compression and motion estimation image compression are proposed. Hadamard image transformation based image compression and Golomb coding gained its importance through dedicated hardware 
architectures. A hardware friendly adaptive decimation based algorithm is also proposed which achieves low bit rate and ended up with low image quality through lossy image compression standards [9]. The existing methods are not relatively suitable for medical images which demand high fidelity rate for compression and decompression techniques [10].

\section{Proposed Work}

The input image is compressed by using DCT method. The harvest of the 2-Dimensional DCT gets in form of sub-windows of $\mathrm{m} \times \mathrm{n}$ arrays. Then the image is converted into differential data by using raster file conversion. By using Golomb-Rice algorithm differential data has been encoded to produce length and code word of the input image. Based on the length of code word obtained the encoded image has been decoded using GolombRice algorithm. The decoded image has been decompressed using the inverse 2-D discrete wavelet transform. The output produced is similar to that of the input image. The proposed hardware architecture is shown in figure 1 .

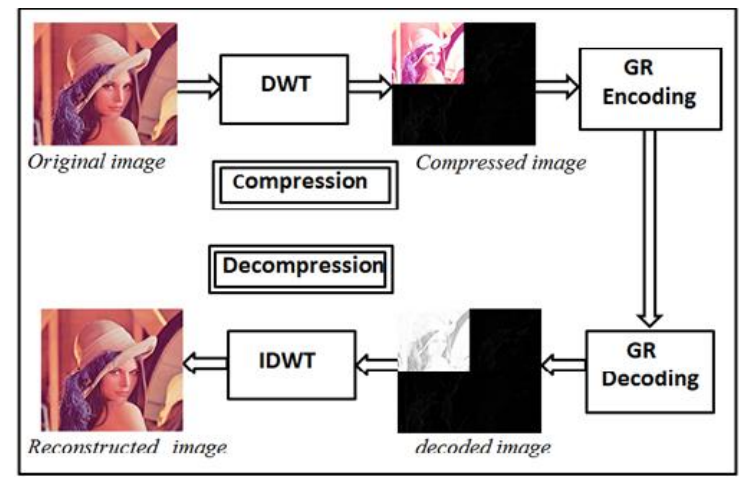

Fig 1 : Hardware Architecture of the Proposed Method

\section{A. Discrete Wavelet Transform}

Wavelet transform is used to determine both the time and the frequency components simultaneously. The different types of DWT are

1-dimensional DWT

2-dimensional DWT

Among these we have utilized2-dimensional DWT. Through this method, the input image which is to be compressed is sent through set of low pass and high pass filters simultaneously. The image from LPF and HPF are down sampled by a factor 2. The block diagram of 2D DWT is shown in fig 2.

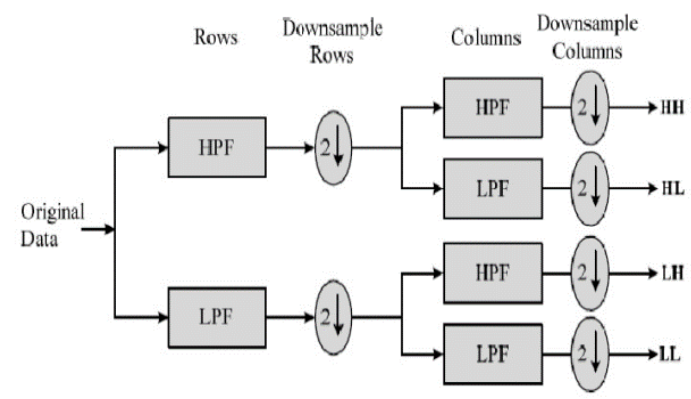

Fig 2: Block of 2-Dimensional DWT 
Four coefficients LL, HL, LH and HH are obtained as outputs. The first letter in 'LL' represents the transformation row-based whereas the second letter represents transformation column-based. The alphabet ' $L$ ' denotes low pass signal and ' $H$ ' denotes high pass signal. Hence, LH signal features a low pass signal in row based transformation and a high pass signal in column based transformation. The LH signal encloses horizontal elements. Similarly, HL and HH enclose vertical and diagonal elements respectively. Raster file is used to convert the image into differential data. Raster graphics are digital images capture as a set of samples of a given space.

\section{B. Golomb-Rice Coder}

The output obtained from 1-D DWT compression is converted into differential data by using raster file conversion. In the Golomb rice encoding the differential data has been encoded then the length has been generated. Based on the generated length the code word has been calculated based on the remainder and quotient bits. Then the code word calculated would be converted into unary code. The unary code obtained from the GR encoding is converted into code word, based on the code word the compressed image has been decoded. The decoded image is applied as an input to 2-D IDWT

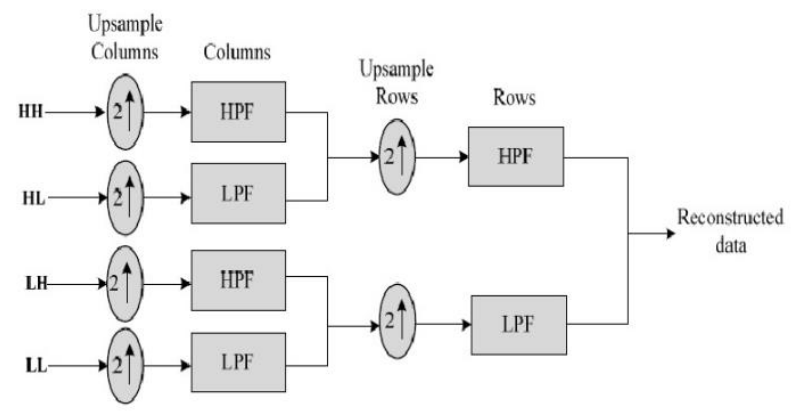

Fig 3: Block of inverse 2-D DWT

The reconstructed image is obtained as a gray image because code word is in the form of 0's and 1's. Then it has been converted into color image by using suitable MATLAB commands.

\section{Experimental Results}

The software implementation of the proposed Golomb-Rice algorithms is performed in MATLAB.PSNR \&compression ratio has been determined and tabulated for simulated images. Lena and man image were used as simulated images. We compared with other techniques and found to be advantageous in picture quality. The simulation results are shown in fig. 4 and the obtained values are tabulated in table 1.

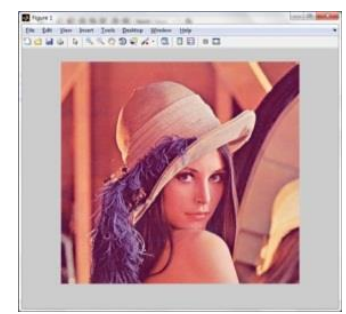

(a)Original image

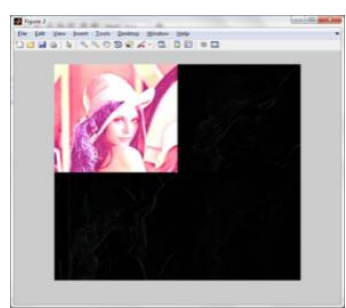

(b) Compressed image 


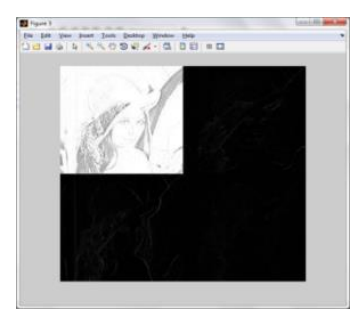

(c) Decoded image

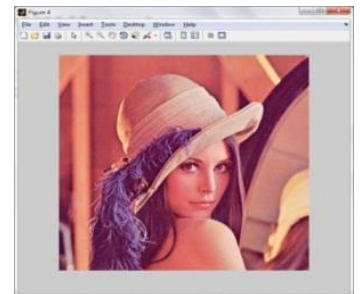

(d) Decompressed image

Fig 4: compression \& decompression of LENA image on Matlab.

Table 1: Comparison of Compression Ratio

\begin{tabular}{|l|l|c|}
\hline METHODS & ALGORITHM & COMPRESSION RATIO (CR) \\
\hline \multirow{3}{*}{ EXISTING } & DDPCM + GR CODING & 1.52 \\
\cline { 2 - 3 } & ARITMETIC CODING & 1.76 \\
\cline { 2 - 3 } & X-MATCH CODING & 1.51 \\
\hline PROPOSED & DWT+GR CODING & 1.90 \\
\hline
\end{tabular}
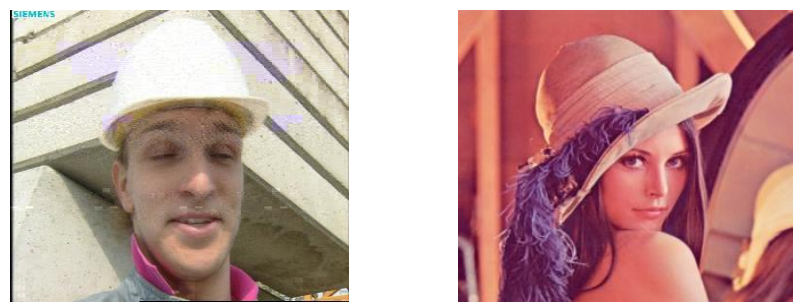

Fig 5: Reconstructed image from MATLAB Simulation

Table 2: Comparison of PSNR and memory size of simulated image

\begin{tabular}{|c|c|c|c|c|c|}
\hline Images & MSE & PSNR & $\begin{array}{c}\text { Original } \\
\text { image } \\
\text { size }\end{array}$ & $\begin{array}{c}\text { Compressed } \\
\text { image size }\end{array}$ & $\begin{array}{c}\text { Reconstructed } \\
\text { image size }\end{array}$ \\
\hline Man & $\begin{array}{c}2.1791 \mathrm{e}+ \\
004\end{array}$ & 4.7 & $297 \mathrm{~kb}$ & $4.4 \mathrm{~kb}$ & $17.6 \mathrm{~kb}$ \\
\hline Lena & $\begin{array}{c}1.5478 \mathrm{e}+ \\
004\end{array}$ & 6.2 & $732 \mathrm{~kb}$ & $14.1 \mathrm{~kb}$ & $37.8 \mathrm{~kb}$ \\
\hline
\end{tabular}

\section{Conclusion}

Image compression and decompression has been performed by using DWT, IDWT and GR coders. The proposed method shows better compression ratio than existing methodologies. The MSE and PSNR values are increased by $44 \%$ and $35 \%$ respectively showing the effectiveness of the proposed methodology. In future it can be implemented using HDL and can be used in Real time applications. 


\section{References}

[1] E. G. Hallnor and S. K. Reinhardt, "A unified compressed memory hier-archy," inProc. 11th Int. Symp. High-Performance Comput. Architect. 2005, pp. 201-212.

[2] S. Roy, R. Kumar, and M. Prvulovic, "Improving system performance with compressed memory," inProc. 15th Int. Parallel Distributed Process. Symp., 2001, pp. 630-636.

[3] J. Ziv and A. Lempel, "Compression of individual sequences via variable rate coding,"IEEE Trans. Inform. Theory, vol. IT-24, no. 5, pp. 530-536, Sep. 1978.

[4] S. W. Golomb, "Run-length codings,"IEEE Trans. Inform. Theory, vol.12, no. 7, pp. 399-401, Jul. 1966.

[5] JPEG LS Image Coding System, document ISO/IEC JTC1/SC29/WG1 N399-WD14495, ISO/IEC, Jul. 1996.

[6] X. Wu and N. D. Memon, "Context-based adaptive lossless image coding,"IEEE Trans. Commun., vol. 45, no. 4, pp. 437-444, Apr. 1997.

[7] Nakar and S. Weiss, "Selective main memory compression by identifying program phase changes," in Proc. 3rd Workshop Memory Performance Issues, 2004, pp. 96-101.

[8] M. Mansour, H. Mouhadjer, A. Alipacha and K. Draoui, "Comparative analysis on image compression techniques for chromosome images," 2013 2nd International Conference on Advances in Biomedical Engineering, Tripoli, 2013, pp. 34-37.

[9] H. M. Mousa, M. A. Ahmad and A. B. El-Sisi, "Image compression ratio enhancement based on conformal mapping," 2013 8th International Conference on Computer Engineering \& Systems (ICCES), Cairo, 2013, pp. 280-285.

[10] Zhang and H. Fang, "Image Compression via Colorization Using Semi-Regular Color Samples," 2013 Data Compression Conference, Snowbird, UT, 2013, pp. 533-533. 